\title{
Performance of the MCP-PMT for the Belle II TOP counter
}

\author{
K. Matsuoka ${ }^{* a}$, S. Hirose ${ }^{b}$, T. $\operatorname{lijima}^{a b}$, K. Inami ${ }^{b}$, Y. Kato ${ }^{a}$, Y. Maeda ${ }^{a}$, R. Mizuno ${ }^{b}$, \\ Y. Sato ${ }^{a}$ and K. Suzuki ${ }^{b}$ \\ ${ }^{a}$ Kobayashi-Maskawa Institute, Nagoya University \\ ${ }^{b}$ Graduate School of Science, Nagoya University \\ E-mail: matsuokadhepl.phys.nagoya-u.ac.jp
}

\begin{abstract}
A square-shaped micro-channel-plate photomultiplier tube (MCP-PMT) was developed for the Belle II time-of-propagation (TOP) counter. Because the TOP counter uses 512 MCP-PMTs in total, mass production of the MCP-PMTs was conducted and their performance was measured systematically. The following results are presented in this paper: the gain, the transit time spread (TTS), the relative collection efficiency and the quantum efficiency (QE). The mass production was successful as the TTS of all the MCP-PMTs was about $30 \mathrm{ps}$ and the average QE at a $360 \mathrm{~nm}$ wavelength was greater than $28 \%$. Degradation of the QE depending on the integrated output charge is a major issue of the MCP-PMT. Study on lifetime extension of the MCP-PMT with atomic layer deposition (ALD) coating is also presented; the lifetime of all the eleven samples with three additional production processes for lifetime extension was measured to be longer than $8.6 \mathrm{C} / \mathrm{cm}^{2}$, or the average lifetime of the normal ALD-MCP-PMTs.
\end{abstract}

International Conference on New Photo-detectors, PhotoDet 2015

6-9 July 2015

Moscow, Troitsk, Russia

\footnotetext{
* Speaker.
} 


\section{Introduction}

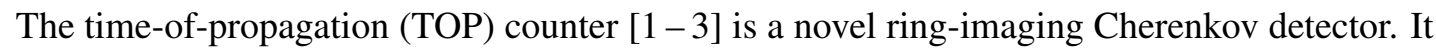
is a key device of particle identification in the Belle II experiment [可] to extend the physics reach toward a new physics. The feature of the TOP counter is that it measures the time of propagation of each Cherenkov photon with a precision better than $50 \mathrm{ps}$ to distinguish a difference of Cherenkov angles. The Cherenkov radiator of the TOP counter is a $2600 \mathrm{~mm}$ long, $450 \mathrm{~mm}$ wide and $20 \mathrm{~mm}$ thick quartz bar, in which Cherenkov photons are totally reflected and propagate toward the end of the bar. Then the photons are expanded by a wedged quartz, called prism, glued on the end of the bar and are detected by two rows of 16 micro-channel-plate photomultiplier tubes (MCP-PMTs). There is no choice other than an MCP-PMT to achieve the required time resolution for single photon detection and we have developed the MCP-PMT [ [ 1 - 1 ] for our application in collaboration with Hamamatsu Photonics K.K. Mass production of the MCP-PMTs was done successfully from 2011 for three years as the Belle II detector is equipped with 16 modules of the TOP counter and hence 512 MCP-PMTs in total.

This paper describes the performance of the mass-produced MCP-PMTs. In Sec. $\square$ the specification of the MCP-PMT is shown, and in Sec. B the actual performance measured for every MCP-PMT is described, including the gain, the transit time spread (TTS), the relative collection efficiency and the quantum efficiency $(\mathrm{QE})$. Though the mass production of the MCP-PMTs finished, our study of the MCP-PMT was still active in terms of extension of the MCP-PMT lifetime, which is explained in Sec. 因.

\section{Specification of the MCP-PMT}

The MCP-PMT for the TOP counter is square-shaped to cover the face of the prism as fully as possible. The outer size is $27.6 \times 27.6 \mathrm{~mm}^{2}$ and the thickness is $13.1 \mathrm{~mm}$ excluding the signal and high voltage pins. The size of the photocathode is $23.0 \times 23.0 \mathrm{~mm}^{2}$. A multi-alkali photocathode, $\mathrm{NaKSbCs}$, is adapted to have a high QE at short wavelengths; the QE peaks around $360 \mathrm{~nm}$. Our requirement for the $\mathrm{QE}$ is at least $24 \%$ at the peak for every MCP-PMT and $28 \%$ or greater on average of all the MCP-PMTs.

The MCP-PMT has enough gain to detect single photons owing to two-stage amplification by a pair of $400 \mu \mathrm{m}$ thick MCPs. The MCP pore size is $10 \mu \mathrm{m}$ in diameter with a 13-degree bias angle. The best time resolution of the MCP-PMT among photon sensors is derived from the electron multiplication in the short transit length inside the tiny MCP pores.

Two types of the MCP-PMT is used for the TOP counter: about a half of the MCP-PMTs with the conventional MCPs were produced firstly, and then the remaining MCP-PMTs were able to be produced using MCPs with atomic layer deposition (ALD) coating [ए]]. The ALD-MCP-PMT has a longer lifetime than the conventional one because the ALD layer prevents outgassing, which is known to be the source of the QE degradation $[$ [ $]$, caused by the secondary electrons impinging on the MCP substrate.

The anode of the MCP-PMT is divided into sixteen $5.275 \times 5.275 \mathrm{~mm}^{2}$ pixels. The performance measurement described below was done for all the sixteen channels. 


\section{Measured performance of the MCP-PMT}

\subsection{Measurement setups}

The gain, the TTS and the relative collection efficiency were measured as a function of the applied high voltage using single photons from a pico-second pulse laser. The wavelength of the laser was approximately $400 \mathrm{~nm}$. The laser was attenuated by two neutral density filters, was collimated by two slits and was divided by a half mirror to be hit on the MCP-PMT and a reference PMT which was used to calibrate the laser intensity. The laser intensity was adjusted to have single photons, for which the hit rate of the MCP-PMT should be several percents. The size of the laser spot on the MCP-PMT was about $1 \mathrm{~mm}$ in diameter. The MCP-PMT was set on a moving stage and the laser spot was located around the center of each anode pixel one-by-one. The signal of the MCP-PMT was amplified by 20, 27 or $35 \mathrm{~dB}$ depending on the high voltage and then it was divided to be read both by a CAMAC ADC and TDC. The signal for the TDC was amplified additionally by $23 \mathrm{~dB}$ to detect even a very small signal when the MCP-PMT gain was as small as $0.5 \times 10^{6}$, and it was discriminated at a threshold of $-20 \mathrm{mV}$. The high voltage dependence was examined at seven different high voltages which correspond to the MCP-PMT gain of roughly $0.5-4 \times 10^{6}$. The high voltage means the one applied to the photocathode. It was divided up by a series of resistors into the four electrodes on the both faces of each MCP. The division ratio was 1:5:5:5:3 in this setup. The measurement was fully automated and the performance of all the MCP-PMTs was comprehended systematically.

The absolute QE of the MCP-PMT $\left(Q E_{P M T}\right)$ was measured by using light from a xenon lamp as follows:

$$
Q E_{P M T}=\frac{I_{P M T}}{I_{P D}} \cdot Q E_{P D},
$$

where $I_{P M T}$ is the photocathode current without amplification by the MCPs measured by a picoammeter, $I_{P D}$ is the current from a reference silicon photodiode measured by the same picoammeter and $Q E_{P D}$ is the absolute $\mathrm{QE}$ of the photodiode which has been measured with a precision of $1.7 \%$ or better. To measure the $\mathrm{QE}$ as a function of the wavelength and the position over the photocathode, the xenon lamp was followed by a monochromator, a sharp cut filter to cut stray light, a variable neutral density filter to tune the light intensity and double slits to make a $\sim 1 \mathrm{~mm}$ diameter light spot on the MCP-PMT. The MCP-PMT was set on a moving stage and thus the light spot scanned the photocathode. The photodiode was fixed on the moving stage alongside of the MCP-PMT and it was moved onto the light spot every time the wavelength was changed. The measurement was also done automatically and systematically for every MCP-PMT with this setup.

\subsection{Results of the measurement}

The gain of the MCP-PMT is defined as the mean of the output charge distribution. As shown in Fig. W (left) the gain $(G)$ increases as an exponential function of the high voltage $(H V)$ :

$$
G=\exp (a \cdot H V+b),
$$

where $a$ and $b$ are parameters to be determined by fitting this function to the data. The gain slope, $a$, is different between the conventional and ALD-MCP-PMTs as shown in Fig. W(right); the ALDMCP-PMT has larger gain than the conventional one at the same high voltage. This is due to the fact that the secondary electron yield of the ALD-MCP is higher than the conventional one. 

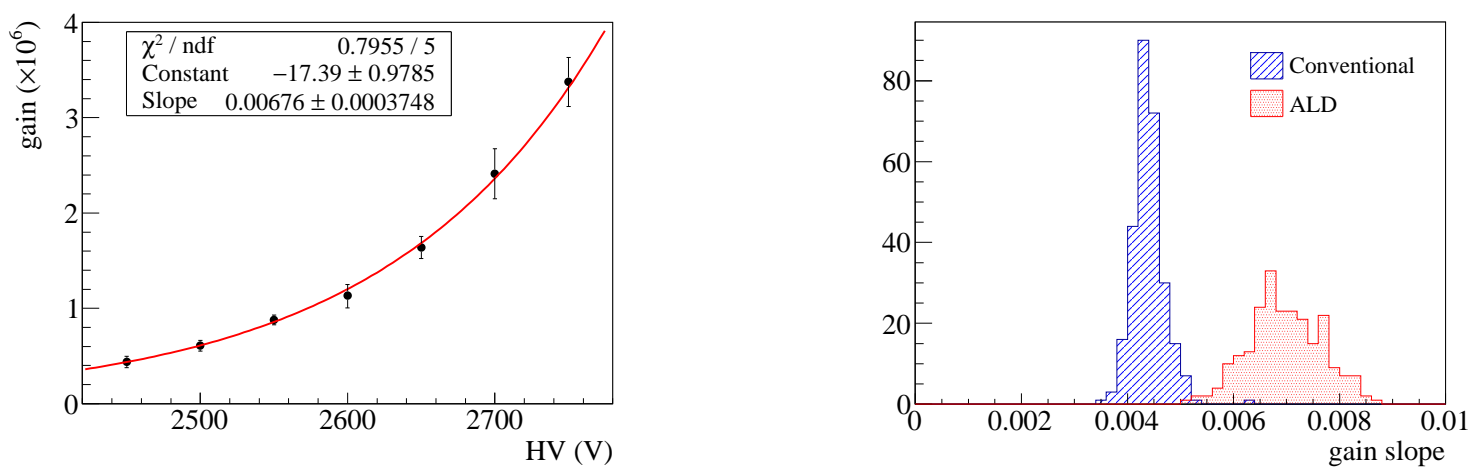

Figure 1: (Left) Gain as a function of the high voltage for one of the MCP-PMT channels. An exponential function fitted to the data is superimposed. (Right) Distribution of the gain slope for the conventional (hatched histogram) and ALD-MCP-PMTs (dotted histogram). Each entry in this plot corresponds to one MCP-PMT or the average of 16 channels.

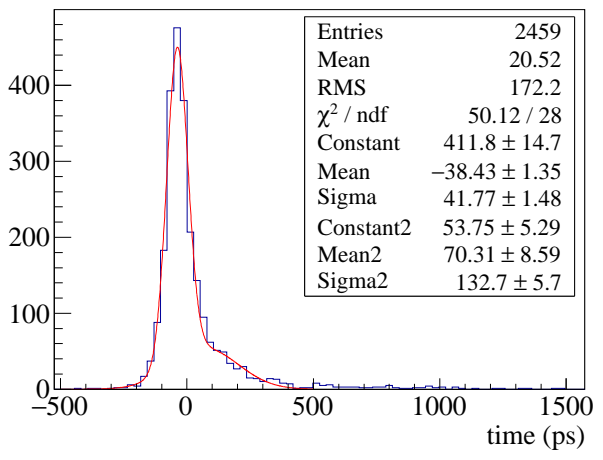

Figure 2: TDC distribution for single photons for one of the conventional-MCP-PMT channels at a high voltage of $3340 \mathrm{~V}$. A double Gaussian fitted to the data is superimposed.

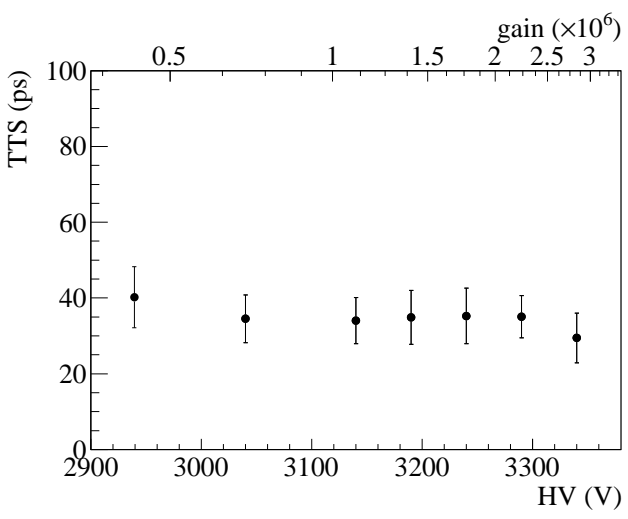

Figure 3: TTS of one of the conventional-MCPPMT channels as a function of the high voltage. Another axis on the top indicates the gain of the MCP-PMT.

A TDC distribution for single photons after time-walk correction is shown in Fig. $\square$ for one of the conventional-MCP-PMT channels. There is a long tail on the right side of the peak. It is due to recoil of the photoelectron on the first MCP surface [12]. A double Gaussian is fitted to the TDC distribution and the standard deviation of the primary Gaussian is defined as the TTS. For this channel, the TTS is $29.5 \mathrm{ps}$ after subtracting the laser pulse width $(\sim 17 \mathrm{ps})$ and the electronics jitter ( $\sim 24.2 \mathrm{ps})$. The TTS of all the other MCP-PMTs was measured to be about $30 \mathrm{ps.} \mathrm{The}$ TTS is nearly independent of the high voltage or the gain down to $0.5 \times 10^{6}$ as shown in Fig. B]. It indicates that the secondary electron yield is nearly constant around $170 \mathrm{~V}$ for the conventional$\mathrm{MCP}$ and around $130 \mathrm{~V}$ for the ALD-MCP, which corresponds to the potential difference between the photocathode and the electrode on the first MCP surface, provided that the TTS depends mainly on the statistics of the secondary electrons at the first multiplication of the photoelectron.

The relative collection efficiency of the MCP-PMT is measured by counting the number of 


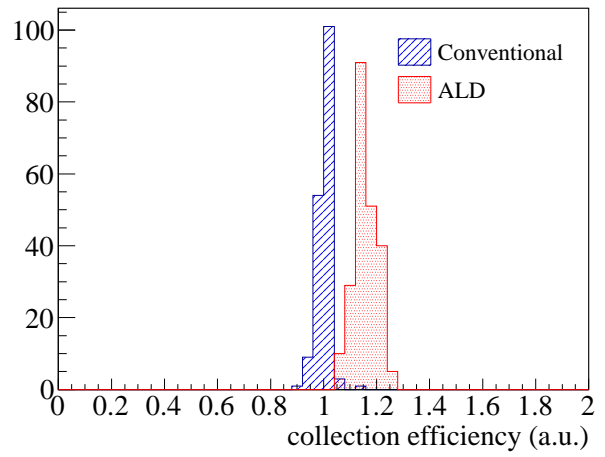

Figure 4: Relative collection efficiency for all the conventional (hatched histogram) and ALDMCP-PMTs (dotted histogram).

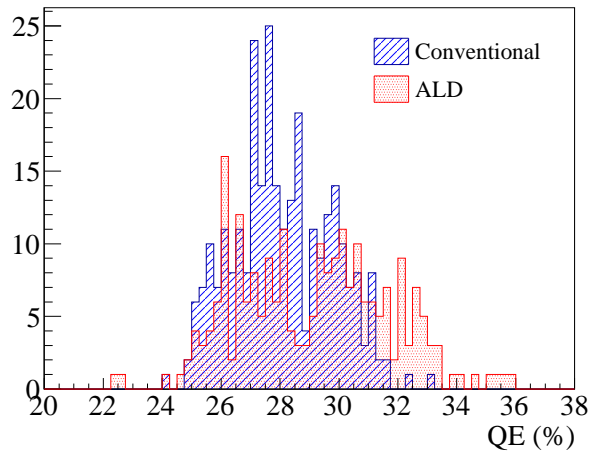

Figure 5: $\mathrm{QE}$ at $360 \mathrm{~nm}$ for all the conventional (hatched histogram) and ALD-MCP-PMTs (dotted histogram) except those whose peak QE is less than $24 \%$.

TDC hits relative to the one of the reference PMT. The PMT-by-PMT collection efficiency is shown in Fig. 1 . The collection efficiency of the ALD-MCP-PMTs is higher by about $15 \%$ than the conventional ones. A comparison of the TDC distributions normalized to the number of photoelectrons between the conventional and ALD-MCP-PMTs reveals that the difference of the collection efficiency appears in the tail made by the recoil photoelectrons on the first MCP surface. Namely the recoil photoelectrons are detectable with higher efficiency for the ALD-MCP as the secondary electron yield of the ALD-MCP is higher than the one of the conventional-MCP.

The $\mathrm{QE}$ was measured at $18 \times 18$ positions including the peripheral non-active area and at 20 wavelengths from $280 \mathrm{~nm}$ to $660 \mathrm{~nm}$. For most of the MCP-PMTs, the QE distributes uniformly over the photocathode and peaks at $360 \mathrm{~nm}$. The QE at $360 \mathrm{~nm}$ for all the MCP-PMTs except those whose peak QE is less than $24 \%$ is shown in Fig. [. The average QE of the conventional and ALD-MCP-PMTs is $28.1 \%$ and $29.1 \%$, respectively, at $360 \mathrm{~nm}$. The maximum QE among them is $35.9 \%$ at $360 \mathrm{~nm}$. The two ALD-MCP-PMTs whose QE is less than $24 \%$ at $360 \mathrm{~nm}$ have the peak $\mathrm{QE}$ at $420 \mathrm{~nm}$.

\section{Extension of the MCP-PMT lifetime}

As the amount of outgassing and the $\mathrm{QE}$ degradation relate to the integrated output charge, the lifetime of the MCP-PMT is defined by the integrated output charge per photocathode area at which the QE drops by $20 \%$ of the beginning. During the mass production, some MCP-PMTs were sampled for the lifetime measurement. The measured lifetime of the conventional and ALD-MCPPMTs was $1.1 \mathrm{C} / \mathrm{cm}^{2}$ and $8.6 \mathrm{C} / \mathrm{cm}^{2}$ on average, respectively. However the output charge due to beam backgrounds in Belle II was predicted by a Monte Carlo simulation as $\sim 3 \mathrm{C} / \mathrm{cm}^{2} / 50 \mathrm{ab}^{-1}$ when the gain of the MCP-PMT was $5 \times 10^{5}$. Therefore the conventional-MCP-PMTs would need to be replaced at some point. Even for the ALD-MCP-PMT, the lifetime is not sufficient for Belle II as the measured lifetime ranged down to $3.2 \mathrm{C} / \mathrm{cm}^{2}$ at the minimum. Thus extension of the ALDMCP-PMT lifetime was demanded. 


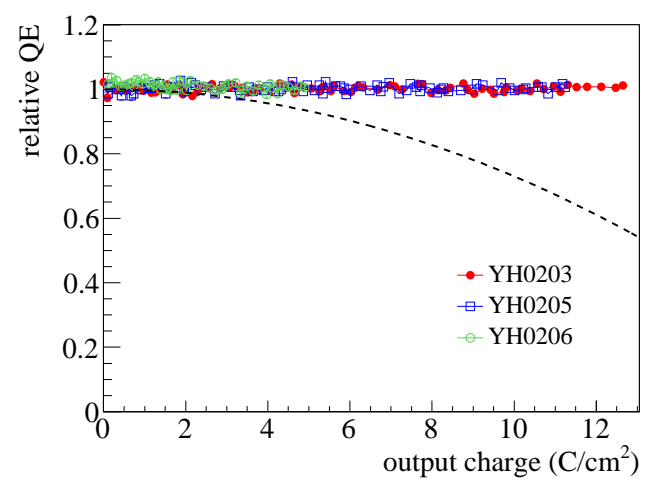

Figure 6: Relative QE as a function of the integrated output charge for three ALD-MCP-PMT samples produced with Method C. The dashed line represents the expected curve for the lifetime of $8.6 \mathrm{C} / \mathrm{cm}^{2}$.

We tried six methods of process in the ALD-MCP-PMT production in order to extend the lifetime and measured the lifetime of several samples for each method. Our criterion for determining whether the method is effective in terms of the lifetime extension is that the measured lifetime is longer than the average lifetime of the normal ALD-MCP-PMTs.

The lifetime is derived from the relative $\mathrm{QE}$ as a function of the integrated output charge. In our setup for the lifetime measurement, eight MCP-PMTs were illuminated at once with a pulsed LED light at $100 \mathrm{kHz}$ and the output charge of each MCP-PMT was measured by a CAMAC ADC. Once a day or so, the LED was turned off to measure the relative QE. The relative QE at $400 \mathrm{~nm}$ was measured by counting the number of TDC hits for single photons from a pico-second pulse laser. The laser intensity was calibrated by a reference PMT, whose high voltage was turned off during the LED illumination. The gain of the MCP-PMTs was set at about $2 \times 10^{6}$.

Among the six methods, three (called Method A, B and C hereafter) were found to be promising; the lifetime of every sample with these three methods (four, four and three samples for Method $\mathrm{A}, \mathrm{B}$ and $\mathrm{C}$, respectively) was longer than $8.6 \mathrm{C} / \mathrm{cm}^{2}$. Especially no $\mathrm{QE}$ degradation was found for the three samples of Method C, which also included Method A and B, up to 4.9, 11.2 and $12.6 \mathrm{C} / \mathrm{cm}^{2}$, respectively, as shown in Fig. 6 .

\section{Conclusion}

Single photon detection with a high efficiency and a time resolution better than $50 \mathrm{ps}$ is one of the most important keys to realize the novel particle identification device, the TOP counter, in Belle II. We succeeded in development and mass production of the square-shaped MCP-PMT which has the QE at $360 \mathrm{~nm}$ greater than $28 \%$ on average and the TTS about $30 \mathrm{ps}$.

Our systematic measurement of all the MCP-PMTs revealed that the high voltage dependence of the gain and the collection efficiency are different between the conventional and ALD-MCPPMTs. This difference originates from the higher secondary electron yield of the ALD-MCP. It can also be seen in the TDC distribution; the tail of the distribution due to the recoil of the photoelectron is larger for the ALD-MCP-PMT. 
We also succeeded in extending the lifetime of the ALD-MCP-PMT by applying the three new methods of production process. The lifetime of all the eleven samples for these methods was measured to be longer than $8.6 \mathrm{C} / \mathrm{cm}^{2}$, or the average lifetime of the normal ALD-MCPPMTs. Especially the three samples produced with all the three methods combined showed no QE degradation up to $4.9,11.2$ and $12.6 \mathrm{C} / \mathrm{cm}^{2}$, respectively.

\section{Acknowledgement}

This work was supported by MEXT Grant-in-Aid for Scientific Research on Innovative Areas "Elucidation of New Hadrons with a Variety of Flavors".

\section{References}

[1] M. Akatsu, et al., Time-of-propagation Cherenkov counter for particle identification, Nucl. Instr. and Meth. A 440 (2000) 124.

[2] T. Oshima, Time-Of-Propagation Counter - A New Cherenkov Ring Imaging Detector, ICFA Instrumentation Bulletin 20 (2000) 2.

[3] K. Matsuoka, For the Belle II PID Group, Design and performance study of the TOP counter, in proceedings of Vienna Conference on Instrumentation 2013, Nucl. Instr. and Meth. A 732 (2013) 357.

[4] T. Abe, et al., Belle II Technical Design Report, KEK-REPORT-2010-1, 2010.

[5] M. Akatsu, et al., MCP-PMT timing property for single photons, Nucl. Instr. and Meth. A 528 (2004) 763.

[6] Y. Enari, et al., Cross-talk of a multi-anode PMT and attainment of a $\sigma \sim 10$ ps TOF counter, Nucl. Instr. and Meth. A 547 (2005) 490.

[7] K. Inami, et al., Cross-talk suppressed multi-anode MCP-PMT, Nucl. Instr. and Meth. A 592 (2008) 247.

[8] N. Kishimoto, et al., Lifetime of MCP-PMT, Nucl. Instr. and Meth. A 564 (2006) 204.

[9] T. Jinno, et al., Lifetime-extended MCP-PMT Nucl. Instr. and Meth. A 629 (2011) 111.

[10] K. Matsuoka, For the Belle II PID Group, Development and production of the MCP-PMT for the Belle II TOP counter, in proceedings of the 8th International Workshop on Ring Imaging Cherenkov Detectors, Nucl. Instr. and Meth. A 766 (2014) 148.

[11] S.M. George, Atomic Layer Deposition: An Overview, Chem. Rev. 2010, 110, 111.

[12] C. Field, et al., Development of photon detectors for a fast focusing DIRC, in proceedings of the 5th international Workshop on Ring Imaging Detectors, Nucl. Instr. and Meth. A 553 (2005) 96. 